\title{
Implementation of Fuzzy C-Means (FCM) Method For Grouping Heart Disorder Patient Data
}

\author{
Sumiati $^{1}$, Yoti Marelita ${ }^{2}$, Akip Suhendar ${ }^{3}$, Riyan Naufal Hay's ${ }^{4}$, Haris Triono Sigit ${ }^{5}$, Ahmad \\ Dedi Jubaedi ${ }^{6}$ \\ \{sumiati82@yahoo.com¹,yotimarelita@gmail.com², akip.suhendar@gmail.com³, \\ riyan.unsera@gmail.com ${ }^{4}$, haris.t.sigit@gmail.com ${ }^{5}$,dedhiest@gmail.com ${ }^{6}$ \} \\ Dept. of Informatics Engineering, Universitas Serang Raya, Serang, Indonesia
}

\begin{abstract}
Heart disease is a fatal disease for human health, resulting in death. Heart disease is a non-communicable disease, but the biggest contributor to mortality, in the amount of 37\% (Indonesia Heart Foundation, 2016). The problems are there no system that is able to read the result of electrocardiogram (ECG), so the ECG result still require a doctor to read the result of electrocardiogram and there is no system that can grouping patients with cardiac abnormalities. This research aims to clustering data on patients with cardiac abnormalities with variable age, heart rate (HR) and bipolar waves (QRS, PQ, QT and QTc) using the Fuzzy C-Means approach. The sample data used in this research were 30 patients. The result of this research indicate that the clustering process stops at 17 th iteration with objective function value 1033148,1702 , there are 2 patients entered into cluster 1 and 28 patients entered into cluster 2 .
\end{abstract}

Keywords: Fuzzy C-Means, Clustering, Heart Disease, Electrocardiogram, Bipolar Waves

\section{Introduction}

Diseases in humans are increasingly increasing and various kinds. Diseases that appear increasingly complex because the pattern of human life is getting worse (M. Cl 2018.). One of the vital organs of humans and is often attacked by diseases, namely the heart. to pump blood throughout the body is the role of the heart. Seeing from heart function that is very important for humans, the risk for people who have heart problems is very large and even leads to death. (al. 2018). [1]. 
Recent research from the Ministry of Health, states that cardiovascular or heart disease is still the deadliest disease in Indonesia. more specifically, the study shows that of the total population at risk of heart disease $39.1 \%$ are aged between 15 and 45 years, contrary to the perception that heart disease is a disease of the elderly. Cardiovascular or heart disease is the most common disease among other non-communicable diseases and accounts for $37 \%$ of the mortality rate due to non-communicable diseases [2].

One way to look at heart abnormalities in a person can be seen from age, heart rate and bipolar waves (QRS, PQ, QT and QTc) using the FCM calculation method to cluster patient data.

\section{Literature Review}

Fuzzy c-means (FCM) technique has been widely used in data clustering. The advantages of FCM such as balancing of individual number of cluster points, drifting of small cluster centers to large neighboring cluster centers, and presence of fuzzy factor, make it more popular. However, early trapping at local minima and high sensitivity to the cluster center initialization are the major limitations of FCM. Clustering is an unsupervised pattern recognition technique used for partitioning the untrained data into various clusters which results in the high degree of similarity in the similar clusters and high degree of dissimilarity in case of dissimilar clusters. In general, there exist two groups of clustering namely hierarchical and partitioning. The first method works in a nested hierarchy fashion by taking an input and producing the corresponding output. On the other hand, the second clustering method is based on the objective function for partitioning the input into a set of fixed number of clusters to produce the output. This clustering process initiates with a random cluster partition and progressively refines the partition for the optimization of the objective function value. Again, the partitioning clustering can be grouped into either hard clusters or soft clusters. FCM is a soft partitioned clustering method introduced by Dunn and Bezdek. FCM is quite popular due to its fuzziness factor in the membership of cluster objects. Although, several attempts have been made to solve the problem of cluster size sensitivity, a very few have been successful in coming close toward the optimal cluster center and achieving the best fitness values of the cluster [3].

The FCM method can be implemented in the fields of education, industry and even medicine, for examples "Penerapan Fuzzy C-Means untuk Penentuan Besar Uang Kuliah Tunggal Mahasiswa Baru"[4], "Penentuan Penerima Beasiswa dengan Algoritma Fuzzy CMeans di Universitas Megos Pak Tulang Bawang”[5], “Clustering Data Nilai Siswa SMA untuk Penentuan Jurusan Menggunakan Algoritma Fuzzy C-Means"[6], "Klastering Industri di Kabupaten Kudus Menggunakan Metode Fuzzy C-Means"[7] and "Deteksi Penyakit Diabetes dengan Metode Fuzzy C-Means dan K-Means Clustering "'[8].

A. Fuzzy C-Means (FCM) algorithm

The FCM algorithm makes use of fuzzy membership function which is used to assign a degree of membership for each class. FCM is able to form new clusters having close 
membership values to existing classes of the data points. The technique of FCM relies on three basic operators such as fuzzy membership function, partition matrix and the objective function. FCM is used to partition a set of $\mathrm{N}$ clusters through minimization of the objective function.

Steps of the FCM algorithm[9]:

1. Input the data that will be cluster $\mathrm{X}$, in the form of a matrix size $\mathrm{n} \times \mathrm{n}(\mathrm{n}=$ number of sample data, $m=$ attribute of each data). $X \_i j=i$ th sample data $(i=$ $1,2, \ldots, n)$, the $j$-attribute $(j=1,2, \ldots, m)$.

2. Specify :

- $\quad$ Number of clusters (c);

- $\quad$ Exponent (w);

- Maximum iteration (MaxIter);

- $\quad$ Error expected value $(\varepsilon)$;

- Initial objective function $\left(P_{0}\right)=0$;

- $\quad$ Initial iteration $(\mathrm{t})=1$

3. Create a random number $\mu \_i k, i=1,2, \ldots, n ; k=1,2, \ldots, c$ as the initial $U$ partition matrix elements.

Calculate each column:

$$
\mathrm{Q}_{\mathrm{i}}=\sum_{\mathrm{k}=1}^{\mathrm{c}} \mu_{\mathrm{i}}
$$

With $\mathrm{j}=1,2, \ldots, \mathrm{m}$

Calculate:

$$
\mu_{\mathrm{ik}}=\frac{\mu_{\mathrm{ik}}}{\mathrm{Q}_{\mathrm{i}}}
$$

4. Calculate the center of the cluster $\mathrm{k}: V_{k j}$, with $\mathrm{k}=1,2, \ldots, \mathrm{c}$; and $\mathrm{j}=1,2, \ldots, \mathrm{m}$

$$
\mathrm{V}_{\mathrm{kj}}=\frac{\sum_{\mathrm{j}=1}^{\mathrm{n}}\left(\left(\mu_{\mathrm{ik}}\right)^{\mathrm{w}} \mathrm{x} \mathrm{X}_{\mathrm{ij}}\right)}{\sum_{\mathrm{j}=1}^{\mathrm{n}}\left(\mu_{\mathrm{ik}}\right)}
$$

5. Calculate the objective function in the initial iteration $(t), p_{t}$ (Yan, 1994)

$$
\mathrm{p}_{\mathrm{t}}=\sum_{\mathrm{i}=1}^{\mathrm{n}} \sum_{\mathrm{k}=1}^{\mathrm{c}}\left(\left[\sum_{\mathrm{j}=1}^{\mathrm{m}}\left(\mathrm{X}_{\mathrm{ij}}-\mathrm{V}_{\mathrm{kj}}\right)^{2}\right]\left(\mu_{\mathrm{ik}}\right)^{\mathrm{w}}\right)
$$

6. Calculate changes of the matrix partition (Yan, 1994)

$$
\mu_{i k}=\frac{\left[\sum_{j=1}^{m}\left(X_{i j}-V_{k j}\right)^{2}\right]^{\frac{-1}{w-1}}}{\sum_{k=1}^{c}\left[\sum_{j=1}^{m}\left(X_{i j}-V_{k j}\right)^{2}\right]^{\frac{-1}{w-1}}}
$$

With : $\mathrm{i}=1,2, \ldots \mathrm{n}$ and $\mathrm{k}=1,2, \ldots \mathrm{c}$.

7. Check the stop condition: 
- if: $(|\mathrm{Pt}-\mathrm{Pt}-1|<\varepsilon)$ or $(\mathrm{t}>$ MaxIter) then stop;

- if not: $\mathrm{t}=\mathrm{t}+1$, repeat step 4

\section{Research Methodology}

This study begins by collecting patient ECG (Electrocardiogram) data, the variables involved are 6 (six) variables consisting of variable X1 (QRS), variable X2 (QTc), variable X3 (PQ), variable X4 (QT), variable X5 (age) and variable X6 (heart rate). the value weight data from each of the above variables will then be obtained with FCM to determine clustering. Where QRS, QTc, PQ and QT are bipolar waves.

FCM tools from MATLAB are used to analyze and cluster data according to the desired amount.

\section{Result and Discussion}

This research examined 30 sample patients data, according to the specified variable. consists of 6 variables 
Table. 1 Sample patients data

\begin{tabular}{|c|c|c|c|c|c|c|}
\hline Patient number & $\mathrm{X} 1$ & $\mathrm{X} 2$ & $\mathrm{X} 3$ & $\mathrm{X} 4$ & X5 & X6 \\
\hline 1 & 63 & 376 & 135 & 376 & 31 & 90 \\
\hline 2 & 93 & 364.702 & 171 & 350 & 26 & 82 \\
\hline 3 & 48 & 354.056 & 144 & 344 & 43 & 82 \\
\hline 4 & 49 & 198.89 & 114 & 270 & 30 & 80 \\
\hline 5 & 65 & 353.999 & 135 & 308 & 35 & 81 \\
\hline 6 & 54 & 378.598 & 137 & 327 & 35 & 87 \\
\hline 7 & 65 & 266.674 & 0 & 215 & 41 & 84 \\
\hline 8 & 41 & 262.85 & 0 & 229 & 48 & 82 \\
\hline 9 & 67 & 372.528 & 67 & 354 & 24 & 80 \\
\hline 10 & 39 & 216.6582 & 0 & 196 & 40 & 98 \\
\hline 11 & 20 & 207.6112 & 135 & 188 & 23 & 97 \\
\hline 12 & 43 & 319.123 & 138 & 276 & 24 & 88 \\
\hline 13 & 55 & -1220.591 & 63 & -110 & 21 & 93 \\
\hline 14 & 44 & 349.669 & 112 & 256 & 22 & 92 \\
\hline 15 & 54 & 207.5406 & 43 & 175 & 23 & 86 \\
\hline 16 & 26 & 323.102 & 108 & 268 & 21 & 85 \\
\hline 17 & 89 & 295.434 & 0 & 223 & 40 & 100 \\
\hline 18 & 58 & 345.9155 & 62 & 289 & 22 & 90 \\
\hline 19 & 83 & -1404 & 78 & -1283 & 22 & 98 \\
\hline 20 & 65 & 297.703 & 153 & 318 & 21 & 102 \\
\hline 21 & 64 & 283.797 & 150 & 305 & 24 & 87 \\
\hline 22 & 68 & 335.036 & 142 & 342 & 23 & 91 \\
\hline 23 & 48 & 417 & 152 & 488 & 33 & 93 \\
\hline 24 & 67 & 424 & 148 & 393 & 45 & 73 \\
\hline 25 & 53 & 423 & 200 & 379 & 23 & 74 \\
\hline 26 & 86 & 424 & 181 & 429 & 35 & 98 \\
\hline 27 & 75 & 350 & 0 & 288 & 27 & 92 \\
\hline 28 & 89 & 229 & 128 & 222 & 30 & 71 \\
\hline 29 & 78 & 352 & 117 & 283 & 21 & 81 \\
\hline 30 & 90 & 406 & 145 & 338 & 22 & 88 \\
\hline
\end{tabular}

FCM calculations start with:

- Determine the initial value 
Table. 2 Initial value

\begin{tabular}{lll}
\hline Initial & Velue & Information \\
\hline number of clusters ( C ) & $2 ;$ & $\mathrm{X}_{1}=$ Umur \\
Exponent ( W ) & $2 ;$ & $\mathrm{X}_{2}=\mathrm{HR}$ \\
Maximum iteration ( MaxIter ) & $100 ;$ & $\mathrm{X}_{3}=\mathrm{QRS}$ \\
Error expected value (X) & $10^{-5} ;$ & $\mathrm{X}_{4}=\mathrm{PQ}$ \\
Initial objective function ( $\left.\mathrm{P}_{0}\right)$ & $0 ;$ & $\mathrm{X}_{5}=\mathrm{QT}$ \\
Initial iteration ( $\mathrm{t}$ ) & $1 ;$ & $\mathrm{X}_{6}=\mathrm{QTc}$ \\
\hline
\end{tabular}

- Create random number

Table. 3 Matrixs U

\begin{tabular}{cc}
\hline Column 1 & Column 2 \\
\hline 0.3 & 0.7 \\
0.2 & 0.8 \\
0.4 & 0.6 \\
0.1 & 0.9 \\
0.5 & 0.5 \\
0.6 & 0.4 \\
0.8 & 0.2 \\
0.7 & 0.3 \\
0.2 & 0.8 \\
0.4 & 0.6 \\
0.1 & 0.9 \\
0.5 & 0.5 \\
0.6 & 0.4 \\
0.8 & 0.2 \\
0.7 & 0.3 \\
0.3 & 0.7 \\
0.4 & 0.6 \\
0.1 & 0.9 \\
0.5 & 0.5 \\
0.6 & 0.4 \\
0.8 & 0.2 \\
0.7 & 0.3 \\
0.3 & 0.7 \\
0.2 & 0.8 \\
0.1 & 0.9 \\
0.5 & 0.5 \\
0.6 & 0.4 \\
0.8 & 0.2 \\
0.7 & 0.3 \\
0.2 & 0.8 \\
\hline &
\end{tabular}


- Calculate cluster center

Table. 4 1st cluster center in final iteration

\begin{tabular}{|c|c|c|c|c|c|c|c|c|c|c|c|c|}
\hline \multirow{2}{*}{$\begin{array}{l}\text { Membership } \\
\text { degree of 1st } \\
\text { cluster }\end{array}$} & \multirow[b]{2}{*}{ xi1 } & \multicolumn{4}{|c|}{ Data on the cluster } & \multirow[b]{2}{*}{ xi6 } & \multirow[b]{2}{*}{$(\operatorname{mil}) 2$} & \multirow[b]{2}{*}{$(\operatorname{mi1} 1) 2 * X i 1$} & \multirow[b]{2}{*}{$(\mathrm{mi} 1) 2 * \mathrm{Xi} 2$} & \multirow[b]{2}{*}{$\left(m_{i 1}\right)^{2 *} X_{i 3}$} & \multirow[b]{2}{*}{$\left(m_{i 1}\right)^{2 *} X_{i 4}$} & \multirow[b]{2}{*}{$\left(m_{i 1}\right)^{2 *} \mathrm{X}_{\mathrm{i} 5}$} \\
\hline & & $\mathrm{xi} 2$ & xi3 & xi4 & xi5 & & & & & & & \\
\hline 0.0022 & 31 & 90 & 63 & 135 & 376 & 376 & $\begin{array}{l}0.000 \\
0\end{array}$ & 0.000144335 & 0.000419038 & 0.000293327 & 0.000628557 & 0.001750648 \\
\hline 0.0022 & 26 & 82 & 93 & 171 & 350 & 364.702 & $\begin{array}{l}0.000 \\
0\end{array}$ & 0.000127854 & 0.000403232 & 0.000457324 & 0.000840886 & 0.001721111 \\
\hline 0.0011 & 43 & 82 & 48 & 144 & 344 & 354.056 & $\begin{array}{l}0.000 \\
0\end{array}$ & 5.09313E-05 & $9.71248 \mathrm{E}-05$ & $5.68535 \mathrm{E}-05$ & 0.000170561 & 0.00040745 \\
\hline 0.0051 & 30 & 80 & 49 & 114 & 270 & 198.89 & $\begin{array}{l}0.000 \\
0\end{array}$ & 0.000765165 & 0.002040441 & 0.00124977 & 0.002907629 & 0.006886489 \\
\hline 0.0004 & 35 & 81 & 65 & 135 & 308 & 353.999 & $\begin{array}{l}0.000 \\
0\end{array}$ & $6.54569 \mathrm{E}-06$ & $1.51486 \mathrm{E}-05$ & $1.21563 \mathrm{E}-05$ & $2.52477 \mathrm{E}-05$ & $5.76021 \mathrm{E}-05$ \\
\hline 0.0011 & 35 & 87 & 54 & 137 & 327 & 378.598 & $\begin{array}{l}0.000 \\
0\end{array}$ & 4.22713E-05 & 0.000105074 & $6.52186 \mathrm{E}-05$ & 0.000165462 & 0.000394935 \\
\hline 0.0064 & 41 & 84 & 65 & 0 & 215 & 266.674 & $\begin{array}{l}0.000 \\
0\end{array}$ & 0.001705421 & 0.003494034 & 0.002703717 & 0 & 0.008943064 \\
\hline 0.0061 & 48 & 82 & 41 & 0 & 229 & 262.85 & $\begin{array}{l}0.000 \\
0\end{array}$ & 0.001783082 & 0.003046099 & 0.001523049 & 0 & 0.008506788 \\
\hline 0.0017 & 24 & 80 & 67 & 67 & 354 & 372.528 & $\begin{array}{l}0.000 \\
0\end{array}$ & $6.71584 \mathrm{E}-05$ & 0.000223861 & 0.000187484 & 0.000187484 & 0.000990587 \\
\hline 0.0106 & 40 & 98 & 39 & 0 & 196 & 216.6582 & $\begin{array}{l}0.000 \\
1\end{array}$ & 0.004471792 & 0.010955891 & 0.004359997 & 0 & 0.021911781 \\
\hline 0.0089 & 23 & 97 & 20 & 135 & 188 & 207.6112 & $\begin{array}{l}0.000 \\
1\end{array}$ & 0.001819341 & 0.007672875 & 0.001582036 & 0.010678743 & 0.014871139 \\
\hline 0.0005 & 24 & 88 & 43 & 138 & 276 & 319.123 & $\begin{array}{l}0.000 \\
0\end{array}$ & $6.141 \mathrm{E}-06$ & $2.2517 \mathrm{E}-05$ & $1.10026 \mathrm{E}-05$ & $3.53107 \mathrm{E}-05$ & $7.06215 \mathrm{E}-05$ \\
\hline 0.8563 & 21 & 93 & 55 & 63 & -110 & - & $\begin{array}{l}0.733 \\
3\end{array}$ & 15.39984058 & 68.19929398 & 40.33291579 & 46.19952173 & $\begin{array}{l}- \\
80.66583159\end{array}$ \\
\hline 0.0008 & 22 & 92 & 44 & 112 & 256 & 349.669 & $\begin{array}{l}0.000 \\
0\end{array}$ & $1.33921 \mathrm{E}-05$ & $5.60035 \mathrm{E}-05$ & $2.67843 \mathrm{E}-05$ & $6.81782 \mathrm{E}-05$ & 0.000155836 \\
\hline 0.0105 & 23 & 86 & 54 & 43 & 175 & 207.5406 & $\begin{array}{l}0.000 \\
1\end{array}$ & 0.002518205 & 0.009415897 & 0.005912307 & 0.004707948 & 0.019160255 \\
\hline 0.0006 & 21 & 85 & 26 & 108 & 268 & 323.102 & $\begin{array}{l}0.000 \\
0\end{array}$ & $8.27527 \mathrm{E}-06$ & $3.34951 \mathrm{E}-05$ & $1.02456 \mathrm{E}-05$ & $4.25585 \mathrm{E}-05$ & 0.000105608 \\
\hline 0.0054 & 40 & 100 & 89 & 0 & 223 & 295.434 & $\begin{array}{l}0.000 \\
0\end{array}$ & 0.001187993 & 0.002969982 & 0.002643284 & 0 & 0.006623061 \\
\hline 0.0007 & 22 & 90 & 58 & 62 & 289 & 345.9155 & $\begin{array}{l}0.000 \\
0\end{array}$ & $1.10381 \mathrm{E}-05$ & 4.51559E-05 & $2.91005 \mathrm{E}-05$ & $3.11074 \mathrm{E}-05$ & 0.000145001 \\
\hline 0.9510 & 22 & 98 & 83 & 78 & $\begin{array}{l}- \\
1283\end{array}$ & -1404 & $\begin{array}{l}0.904 \\
4\end{array}$ & 19.89648727 & 88.62980692 & 75.06402014 & 70.54209122 & $\begin{array}{l}- \\
1160.326962\end{array}$ \\
\hline 0.0009 & 21 & 102 & 65 & 153 & 318 & 297.703 & $\begin{array}{l}0.000 \\
0\end{array}$ & $1.70418 \mathrm{E}-05$ & $8.27745 \mathrm{E}-05$ & $5.27485 \mathrm{E}-05$ & 0.000124162 & 0.000258062 \\
\hline 0.0010 & 24 & 87 & 64 & 150 & 305 & 283.797 & $\begin{array}{l}0.000 \\
0\end{array}$ & $2.18802 \mathrm{E}-05$ & 7.93156E-05 & $5.83471 \mathrm{E}-05$ & 0.000136751 & 0.00027806 \\
\hline 0.0008 & 23 & 91 & 68 & 142 & 342 & 335.036 & $\begin{array}{l}0.000 \\
0\end{array}$ & $1.39707 \mathrm{E}-05$ & $5.52753 \mathrm{E}-05$ & $4.13046 \mathrm{E}-05$ & $8.62538 \mathrm{E}-05$ & 0.000207738 \\
\hline 0.0099 & 33 & 93 & 48 & 152 & 488 & 417 & $\begin{array}{l}0.000 \\
1\end{array}$ & 0.00322067 & 0.009076433 & 0.00468461 & 0.0148346 & 0.047626873 \\
\hline 0.0046 & 45 & 73 & 67 & 148 & 393 & 424 & $\begin{array}{l}0.000 \\
0\end{array}$ & 0.000967103 & 0.001568856 & 0.001439909 & 0.003180693 & 0.00844603 \\
\hline 0.0056 & 23 & 74 & 53 & 200 & 379 & 423 & $\begin{array}{l}0.000 \\
0\end{array}$ & 0.000720101 & 0.002316847 & 0.001659363 & 0.006261748 & 0.011866013 \\
\hline 0.0072 & 35 & 98 & 86 & 181 & 429 & 424 & $\begin{array}{l}0.000 \\
1\end{array}$ & 0.001814217 & 0.005079808 & 0.004457791 & 0.009382095 & 0.02223712 \\
\hline
\end{tabular}




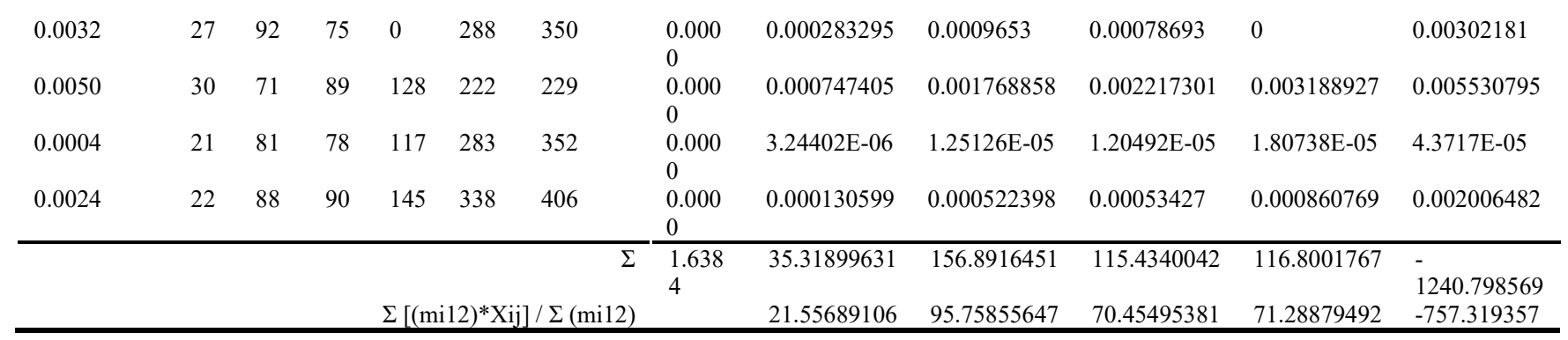

Table. 5 2sd cluster center in final iteration

\begin{tabular}{|c|c|c|c|c|c|c|c|c|c|c|c|c|c|}
\hline $\begin{array}{l}\text { Members } \\
\text { hip } \\
\text { degree of } \\
2 \text { st } \\
\text { cluster }\end{array}$ & & & Dat & on the & uster & & $\left(\mu_{\mathrm{i} 1}\right)^{2}$ & $\begin{array}{c}\left(\mu_{\mathrm{i} 1}\right)^{2 *} \\
\mathrm{X}_{\mathrm{i} 1}\end{array}$ & $\begin{array}{c}\left(\mu_{\mathrm{i} 1}\right)^{2} \\
* \mathrm{X}_{\mathrm{i} 2}\end{array}$ & $\begin{array}{l}\left(\mu_{\mathrm{i} 1}\right)^{2} \\
* \mathrm{X}_{\mathrm{i} 3}\end{array}$ & $\begin{array}{c}\left(\mu_{\mathrm{i} 1}\right)^{2 *} \mathrm{X}_{\mathrm{i}} \\
4\end{array}$ & $\underset{5}{\left(\mu_{\mathrm{i} 1}\right)^{2 *} \mathrm{X}_{\mathrm{i}}}$ & $\begin{array}{c}\left(\mu_{i 1}\right)^{2 *} X_{i} \\
6\end{array}$ \\
\hline 0.9978 & 31 & 90 & 63 & 135 & 376 & 376 & 0.9957 & $\begin{array}{l}30.8663 \\
6251\end{array}$ & $\begin{array}{l}89.612 \\
02018\end{array}$ & $\begin{array}{l}62.728 \\
41412\end{array}$ & $\begin{array}{l}134.4180 \\
303\end{array}$ & $\begin{array}{l}374.3791 \\
065\end{array}$ & $\begin{array}{l}374.3791 \\
065\end{array}$ \\
\hline 0.9978 & 26 & 82 & 93 & 171 & 350 & 364.702 & 0.9956 & $\begin{array}{l}25.8848 \\
1604\end{array}$ & $\begin{array}{l}81.636 \\
72752\end{array}$ & $\begin{array}{l}92.587 \\
99584\end{array}$ & $\begin{array}{l}170.2424 \\
44\end{array}$ & $\begin{array}{l}348.4494 \\
467\end{array}$ & $\begin{array}{l}363.0863 \\
146\end{array}$ \\
\hline 0.9989 & 43 & 82 & 48 & 144 & 344 & 354.056 & 0.9978 & $\begin{array}{l}42.9064 \\
551\end{array}$ & $\begin{array}{l}81.821 \\
61204\end{array}$ & $\begin{array}{l}47.895 \\
57778\end{array}$ & $\begin{array}{l}143.6867 \\
333\end{array}$ & $\begin{array}{l}343.2516 \\
408\end{array}$ & $\begin{array}{l}353.2857 \\
643\end{array}$ \\
\hline 0.9949 & 30 & 80 & 49 & 114 & 270 & 198.89 & 0.9899 & $\begin{array}{l}29.6977 \\
4725\end{array}$ & $\begin{array}{l}79.193 \\
99266\end{array}$ & $\begin{array}{l}48.506 \\
32051\end{array}$ & $\begin{array}{l}112.8514 \\
395\end{array}$ & $\begin{array}{l}267.2797 \\
252\end{array}$ & $\begin{array}{l}196.8861 \\
65\end{array}$ \\
\hline 0.9996 & 35 & 81 & 65 & 135 & 308 & 353.999 & 0.9991 & $\begin{array}{l}34.9697 \\
3449\end{array}$ & $\begin{array}{l}80.929 \\
95697\end{array}$ & $\begin{array}{l}64.943 \\
79263\end{array}$ & $\begin{array}{l}134.8832 \\
616\end{array}$ & $\begin{array}{l}307.7336 \\
635\end{array}$ & $\begin{array}{l}353.6928 \\
869\end{array}$ \\
\hline 0.9989 & 35 & 87 & 54 & 137 & 327 & 378.598 & 0.9978 & $\begin{array}{l}34.9231 \\
1381\end{array}$ & $\begin{array}{l}86.808 \\
88291\end{array}$ & $\begin{array}{l}53.881 \\
3756\end{array}$ & $\begin{array}{l}136.6990 \\
455\end{array}$ & $\begin{array}{l}326.2816 \\
634\end{array}$ & $\begin{array}{l}377.7663 \\
155\end{array}$ \\
\hline 0.9936 & 41 & 84 & 65 & 0 & 215 & 266.674 & 0.9871 & $\begin{array}{l}40.4728 \\
49\end{array}$ & $\begin{array}{l}82.919 \\
98331\end{array}$ & $\begin{array}{l}64.164 \\
2728\end{array}$ & 0 & $\begin{array}{l}212.2356 \\
716\end{array}$ & $\begin{array}{l}263.2452 \\
813\end{array}$ \\
\hline 0.9939 & 48 & 82 & 41 & 0 & 229 & 262.85 & 0.9878 & $\begin{array}{l}47.4166 \\
7471\end{array}$ & $\begin{array}{l}81.003 \\
48597\end{array}$ & $\begin{array}{l}40.501 \\
74298\end{array}$ & 0 & $\begin{array}{l}226.2170 \\
523\end{array}$ & $\begin{array}{l}259.6556 \\
864\end{array}$ \\
\hline 0.9983 & 24 & 80 & 67 & 67 & 354 & 372.528 & 0.9967 & $\begin{array}{l}23.9197 \\
7265\end{array}$ & $\begin{array}{l}79.732 \\
5755\end{array}$ & $\begin{array}{l}66.776 \\
03198\end{array}$ & $\begin{array}{l}66.77603 \\
198\end{array}$ & $\begin{array}{l}352.8166 \\
466\end{array}$ & $\begin{array}{l}371.2827 \\
111\end{array}$ \\
\hline 0.9894 & 40 & 98 & 39 & 0 & 196 & 216.6582 & 0.9790 & $\begin{array}{l}39.1586 \\
0731\end{array}$ & $\begin{array}{l}95.938 \\
5879\end{array}$ & $\begin{array}{l}38.179 \\
64212\end{array}$ & 0 & $\begin{array}{l}191.8771 \\
758\end{array}$ & $\begin{array}{l}212.1008 \\
343\end{array}$ \\
\hline 0.9911 & 23 & 97 & 20 & 135 & 188 & 207.6112 & 0.9823 & $\begin{array}{l}22.5926 \\
9905\end{array}$ & $\begin{array}{l}95.282 \\
25253\end{array}$ & $\begin{array}{l}19.645 \\
82526\end{array}$ & $\begin{array}{l}132.6093 \\
205\end{array}$ & $\begin{array}{l}184.6707 \\
575\end{array}$ & $\begin{array}{l}203.9346 \\
679\end{array}$ \\
\hline 0.9995 & 24 & 88 & 43 & 138 & 276 & 319.123 & 0.9990 & $\begin{array}{l}23.9757 \\
2578\end{array}$ & $\begin{array}{l}87.910 \\
99453\end{array}$ & $\begin{array}{l}42.956 \\
50869\end{array}$ & $\begin{array}{l}137.8604 \\
232\end{array}$ & $\begin{array}{l}275.7208 \\
465\end{array}$ & $\begin{array}{l}318.8002 \\
308\end{array}$ \\
\hline 0.1437 & 21 & 93 & 55 & 63 & -110 & -1220.59 & 0.0206 & $\begin{array}{l}0.43337 \\
5523\end{array}$ & $\begin{array}{l}1.9192 \\
34461\end{array}$ & $\begin{array}{l}1.1350 \\
31133\end{array}$ & $\begin{array}{l}1.300126 \\
57\end{array}$ & $\begin{array}{l}- \\
2.270062 \\
265\end{array}$ & $\begin{array}{l}- \\
25.18925 \\
064\end{array}$ \\
\hline 0.9992 & 22 & 92 & 44 & 112 & 256 & 349.669 & 0.9984 & $\begin{array}{l}21.9656 \\
8398\end{array}$ & $\begin{array}{l}91.856 \\
49666\end{array}$ & $\begin{array}{l}43.931 \\
36797\end{array}$ & $\begin{array}{l}111.8253 \\
003\end{array}$ & $\begin{array}{l}255.6006 \\
864\end{array}$ & $\begin{array}{l}349.1235 \\
797\end{array}$ \\
\hline 0.9895 & 23 & 86 & 54 & 43 & 175 & 207.5406 & 0.9792 & $\begin{array}{l}22.5211 \\
9206\end{array}$ & $\begin{array}{l}84.209 \\
67466\end{array}$ & $\begin{array}{l}52.875 \\
84223\end{array}$ & $\begin{array}{l}42.10483 \\
733\end{array}$ & $\begin{array}{l}171.3568 \\
961\end{array}$ & $\begin{array}{l}203.2200 \\
745\end{array}$ \\
\hline 0.9994 & 21 & 85 & 26 & 108 & 268 & 323.102 & 0.9987 & $\begin{array}{l}20.9736 \\
4309\end{array}$ & $\begin{array}{l}84.893 \\
31729\end{array}$ & $\begin{array}{l}25.967 \\
36764\end{array}$ & $\begin{array}{l}107.8644 \\
502\end{array}$ & $\begin{array}{l}267.6636 \\
357\end{array}$ & $\begin{array}{l}322.6964 \\
777\end{array}$ \\
\hline 0.9946 & 40 & 100 & 89 & 0 & 223 & 295.434 & 0.9891 & $\begin{array}{l}39.5652 \\
0763\end{array}$ & $\begin{array}{l}98.913 \\
01907\end{array}$ & $\begin{array}{l}88.032 \\
58697\end{array}$ & 0 & $\begin{array}{l}220.5760 \\
325\end{array}$ & $\begin{array}{l}292.2226 \\
888\end{array}$ \\
\hline
\end{tabular}




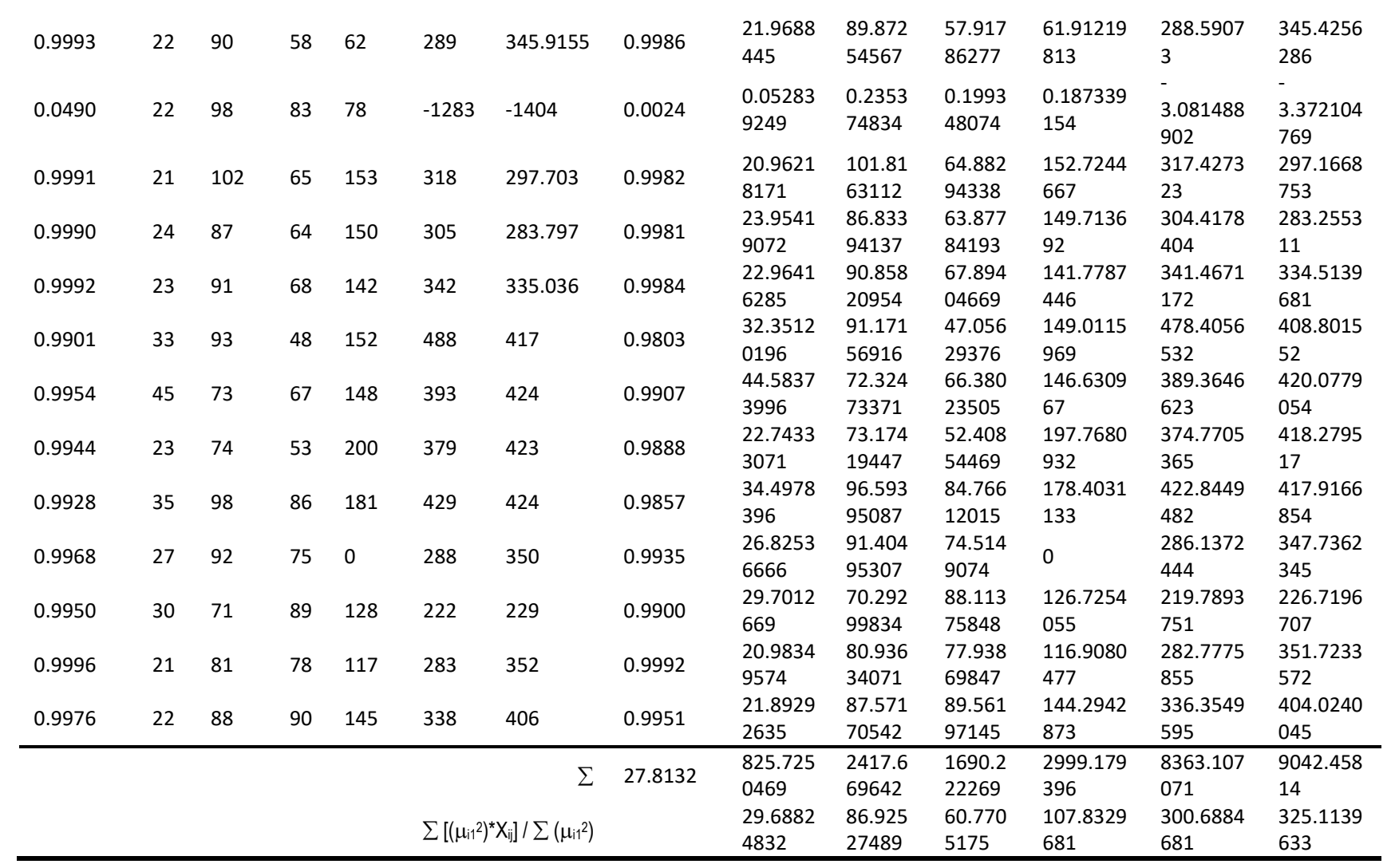

Table. 6 Cluster Center Results in Final Iteration

\begin{tabular}{rrrrrrr}
\hline \multirow{2}{*}{$\mathrm{V}_{\mathrm{kj}}$} & 21,55689106 & 95,75855647 & 70,45495381 & 71,28879492 & $-757,319357$ & $-1321,188519$ \\
& 29,68824832 & 86,92527489 & 60,7705175 & 107,8329681 & 300,6884681 & 325,1139633 \\
\hline
\end{tabular}

- Calculating objective function 
Table. 7 Results of Objective Functions in

\begin{tabular}{|c|c|c|c|c|}
\hline \multicolumn{2}{|c|}{ Squared membershio degree of $i$ data } & \multirow{2}{*}{$\begin{array}{l}\mathrm{X} 2 \\
\mathrm{~L} 1\end{array}$} & \multirow{2}{*}{$\begin{array}{l}\mathrm{X} 3 \\
\mathrm{~L} 2\end{array}$} & \multirow{2}{*}{$\mathrm{L} 1+\mathrm{L} 2$} \\
\hline $\mathrm{m}_{\mathrm{i} 1}{ }^{2}$ & $\mathrm{~m}_{\mathrm{i} 2}{ }^{2}$ & & & \\
\hline 0.0000 & 0.9957 & 19.41123002 & 8976.544002 & 8995.955232 \\
\hline 0.0000 & 0.9956 & 20.0585322 & 9025.353185 & 9045.411717 \\
\hline 0.0000 & 0.9978 & 4.768339106 & 4376.589796 & 4381.358135 \\
\hline 0.0000 & 0.9899 & 85.91859452 & 16926.63194 & 17012.55054 \\
\hline 0.0000 & 0.9991 & 0.737914038 & 1705.587428 & 1706.325342 \\
\hline 0.0000 & 0.9978 & 4.915394425 & 4467.778644 & 4472.694039 \\
\hline 0.0000 & 0.9871 & 144.4342422 & 22250.28769 & 22394.72193 \\
\hline 0.0000 & 0.9878 & 129.6019305 & 21134.44027 & 21264.0422 \\
\hline 0.0000 & 0.9967 & 11.484064 & 6853.676291 & 6865.160355 \\
\hline 0.0001 & 0.9790 & 366.7100905 & 34315.8762 & 34682.58629 \\
\hline 0.0001 & 0.9823 & 256.0893679 & 28537.62961 & 28793.71898 \\
\hline 0.0000 & 0.9990 & 0.963021518 & 1902.837879 & 1903.8009 \\
\hline 0.7333 & 0.0206 & 314932.3793 & 52831.04629 & 367763.4256 \\
\hline 0.0000 & 0.9984 & 2.325942334 & 2978.831717 & 2981.15766 \\
\hline 0.0001 & 0.9792 & 351.1692074 & 33209.77844 & 33560.94764 \\
\hline 0.0000 & 0.9987 & 1.48104149 & 2357.830062 & 2359.311103 \\
\hline 0.0000 & 0.9891 & 106.3336456 & 19405.28195 & 19511.6156 \\
\hline 0.0000 & 0.9986 & 1.943857895 & 2742.334952 & 2744.27881 \\
\hline 0.9044 & 0.0024 & 256307.9224 & 13208.53961 & 269516.462 \\
\hline 0.0000 & 0.9982 & 3.070665086 & 3405.588247 & 3408.658912 \\
\hline 0.0000 & 0.9981 & 3.383055713 & 3539.761322 & 3543.144378 \\
\hline 0.0000 & 0.9984 & 2.403329954 & 3081.268486 & 3083.671816 \\
\hline 0.0001 & 0.9803 & 446.9193219 & 44792.03954 & 45238.95886 \\
\hline 0.0000 & 0.9907 & 94.04282422 & 20191.9036 & 20285.94642 \\
\hline 0.0000 & 0.9888 & 136.2168834 & 24208.11029 & 24344.32718 \\
\hline 0.0001 & 0.9857 & 231.4682346 & 31918.48842 & 32149.95666 \\
\hline 0.0000 & 0.9935 & 40.82287342 & 12561.94041 & 12602.76329 \\
\hline 0.0000 & 0.9900 & 83.86865977 & 16718.93559 & 16802.80425 \\
\hline 0.0000 & 0.9992 & 0.60001835 & 1526.024681 & 1526.624699 \\
\hline \multirow[t]{2}{*}{0.0000} & 0.9951 & 24.86601574 & 10180.92371 & 10205.78973 \\
\hline & & \multicolumn{2}{|c|}{ Objective function } & 1033148.17 \\
\hline
\end{tabular}


Final Iteration

- Converson of the matrix

Table. 8 Results of Changes in the Matrix on Iteration

\begin{tabular}{lccll}
\hline$\sum_{k=1}^{3}\left[\sum_{j=1}^{6}\left(X_{i j}-V_{k j}\right)^{2}\right]^{-1}$ & {$\left[\sum_{j=1}^{6}\left(X_{i j}-V_{1 j}\right)^{2}\right]$} & {$\left[\sum_{j=1}^{6}\left(X_{i j}-V_{2 j}\right)^{2}\right]$} & \multicolumn{1}{l}{$\mathrm{m}_{\mathrm{i} 1}$} & \multicolumn{1}{l}{$\mathrm{m}_{\mathrm{i} 2}$} \\
$\mathrm{~L} 1$ & $\mathrm{~L} 2$ & $\mathrm{LT}=\mathrm{L} 1+\mathrm{L} 2$ & $\mathrm{~L} 1 / \mathrm{LT}$ & $\mathrm{L} 2 / \mathrm{LT}$ \\
\hline $2.39860 \mathrm{E}-07$ & 0.000110921 & $1.11161 \mathrm{E}-04$ & $2.15777 \mathrm{E}-03$ & $9.97842 \mathrm{E}-01$ \\
$2.45156 \mathrm{E}-07$ & 0.000110308 & $1.10553 \mathrm{E}-04$ & $2.21753 \mathrm{E}-03$ & $9.97782 \mathrm{E}-01$ \\
$2.48399 \mathrm{E}-07$ & 0.000227991 & $2.28240 \mathrm{E}-04$ & $1.08832 \mathrm{E}-03$ & $9.98912 \mathrm{E}-01$ \\
$2.96857 \mathrm{E}-07$ & $5.84833 \mathrm{E}-05$ & $5.87801 \mathrm{E}-05$ & $5.05029 \mathrm{E}-03$ & $9.94950 \mathrm{E}-01$ \\
$2.53444 \mathrm{E}-07$ & 0.000585801 & $5.86055 \mathrm{E}-04$ & $4.32458 \mathrm{E}-04$ & $9.99568 \mathrm{E}-01$ \\
$2.45708 \mathrm{E}-07$ & 0.000223333 & $2.23579 \mathrm{E}-04$ & $1.09898 \mathrm{E}-03$ & $9.98901 \mathrm{E}-01$ \\
$2.87990 \mathrm{E}-07$ & $4.43654 \mathrm{E}-05$ & $4.46534 \mathrm{E}-05$ & $6.44946 \mathrm{E}-03$ & $9.93551 \mathrm{E}-01$ \\
$2.86628 \mathrm{E}-07$ & $4.67411 \mathrm{E}-05$ & $4.70277 \mathrm{E}-05$ & $6.09487 \mathrm{E}-03$ & $9.93905 \mathrm{E}-01$ \\
$2.43665 \mathrm{E}-07$ & 0.000145419 & $1.45663 \mathrm{E}-04$ & $1.67280 \mathrm{E}-03$ & $9.98327 \mathrm{E}-01$ \\
$3.04859 \mathrm{E}-07$ & $2.85281 \mathrm{E}-05$ & $2.88329 \mathrm{E}-05$ & $1.05733 \mathrm{E}-02$ & $9.89427 \mathrm{E}-01$ \\
$3.08884 \mathrm{E}-07$ & $3.44209 \mathrm{E}-05$ & $3.47298 \mathrm{E}-05$ & $8.89391 \mathrm{E}-03$ & $9.91106 \mathrm{E}-01$ \\
$2.65700 \mathrm{E}-07$ & 0.000524999 & $5.25265 \mathrm{E}-04$ & $5.05840 \mathrm{E}-04$ & $9.99494 \mathrm{E}-01$ \\
$2.32852 \mathrm{E}-06$ & $3.90621 \mathrm{E}-07$ & $2.71914 \mathrm{E}-06$ & $8.56344 \mathrm{E}-01$ & $1.43656 \mathrm{E}-01$ \\
$2.61715 \mathrm{E}-07$ & 0.000335178 & $3.35440 \mathrm{E}-04$ & $7.80213 \mathrm{E}-04$ & $9.99220 \mathrm{E}-01$ \\
$3.11779 \mathrm{E}-07$ & $2.94848 \mathrm{E}-05$ & $2.97965 \mathrm{E}-05$ & $1.04636 \mathrm{E}-02$ & $9.89536 \mathrm{E}-01$ \\
$2.66070 \mathrm{E}-07$ & 0.000423586 & $4.23853 \mathrm{E}-04$ & $6.27742 \mathrm{E}-04$ & $9.99372 \mathrm{E}-01$ \\
$2.79308 \mathrm{E}-07$ & $5.09722 \mathrm{E}-05$ & $5.12515 \mathrm{E}-05$ & $5.44975 \mathrm{E}-03$ & $9.94550 \mathrm{E}-01$ \\
$2.58111 \mathrm{E}-07$ & 0.000364136 & $3.64394 \mathrm{E}-04$ & $7.08330 \mathrm{E}-04$ & $9.99292 \mathrm{E}-01$ \\
$3.52851 \mathrm{E}-06$ & $1.81836 \mathrm{E}-07$ & $3.71035 \mathrm{E}-06$ & $9.50992 \mathrm{E}-01$ & $4.90077 \mathrm{E}-02$ \\
$2.64280 \mathrm{E}-07$ & 0.000293106 & $2.93371 \mathrm{E}-04$ & $9.00840 \mathrm{E}-04$ & $9.99099 \mathrm{E}-01$ \\
$2.69482 \mathrm{E}-07$ & 0.000281966 & $2.82235 \mathrm{E}-04$ & $9.54814 \mathrm{E}-04$ & $9.99045 \mathrm{E}-01$ \\
$2.52741 \mathrm{E}-07$ & 0.000324036 & $3.24289 \mathrm{E}-04$ & $7.79371 \mathrm{E}-04$ & $9.99221 \mathrm{E}-01$ \\
\hline
\end{tabular}

- Check the stop condition

$(|\mathrm{Pt}-\mathrm{Pt}-1|<\varepsilon)$ or $(\mathrm{t}>$ MaxIter $)$ it will be stop;

$(|1033148,170248-1033148,170231|<\varepsilon)=$ true OR $(17>100)=$ false.

The calculation stops, because the results have shown the error results stably.

The test results show that the clustering process stops at the 17 th iteration with an objective function value of 1033148,1702 . And produce 2 (two) clusters. 
- Result of cluster

Table. 9 Degree of Membership of Each Data in Each Cluster

\begin{tabular}{|c|c|c|c|c|c|}
\hline \multirow[t]{2}{*}{ Data } & \multicolumn{2}{|c|}{$\begin{array}{l}\text { Membership degree of Data }(m) \text { in } \\
\text { Cluster }\end{array}$} & \multirow{2}{*}{$\begin{array}{c}\text { Cluster Data based on Membership } \\
\text { Degree }\end{array}$} & \multirow{2}{*}{$\mathrm{C} 1$} & \multirow{2}{*}{$\mathrm{C} 2$} \\
\hline & 1 & 2 & & & \\
\hline 1 & 0.0022 & 0.9978 & 0.9978 & & $*$ \\
\hline 2 & 0.0022 & 0.9978 & 0.9978 & & $*$ \\
\hline 3 & 0.0011 & 0.9989 & 0.9989 & & $*$ \\
\hline 4 & 0.0051 & 0.9949 & 0.9949 & & $*$ \\
\hline 5 & 0.0004 & 0.9996 & 0.9996 & & $*$ \\
\hline 6 & 0.0011 & 0.9989 & 0.9989 & & $*$ \\
\hline 7 & 0.0064 & 0.9936 & 0.9936 & & $*$ \\
\hline 8 & 0.0061 & 0.9939 & 0.9939 & & $*$ \\
\hline 9 & 0.0017 & 0.9983 & 0.9983 & & $*$ \\
\hline 10 & 0.0106 & 0.9894 & 0.9894 & & $*$ \\
\hline 11 & 0.0089 & 0.9911 & 0.9911 & & $*$ \\
\hline 12 & 0.0005 & 0.9995 & 0.9995 & & $*$ \\
\hline 13 & 0.8563 & 0.1437 & 0.8563 & $*$ & \\
\hline 14 & 0.0008 & 0.9992 & 0.9992 & & $*$ \\
\hline 15 & 0.0105 & 0.9895 & 0.9895 & & $*$ \\
\hline 16 & 0.0006 & 0.9994 & 0.9994 & & $*$ \\
\hline 17 & 0.0054 & 0.9946 & 0.9946 & & $*$ \\
\hline 18 & 0.0007 & 0.9993 & 0.9993 & & $*$ \\
\hline 19 & 0.9510 & 0.0490 & 0.9510 & $*$ & \\
\hline 20 & 0.0009 & 0.9991 & 0.9991 & & $*$ \\
\hline 21 & 0.0010 & 0.9990 & 0.9990 & & $*$ \\
\hline 22 & 0.0008 & 0.9992 & 0.9992 & & $*$ \\
\hline 23 & 0.0099 & 0.9901 & 0.9901 & & $*$ \\
\hline 24 & 0.0046 & 0.9954 & 0.9954 & & $*$ \\
\hline 25 & 0.0056 & 0.9944 & 0.9944 & & $*$ \\
\hline 26 & 0.0072 & 0.9928 & 0.9928 & & $*$ \\
\hline 27 & 0.0032 & 0.9968 & 0.9968 & & $*$ \\
\hline 28 & 0.0050 & 0.9950 & 0.9950 & & $*$ \\
\hline 29 & 0.0004 & 0.9996 & 0.9996 & & $*$ \\
\hline 30 & 0.0024 & 0.9976 & 0.9976 & & * \\
\hline
\end{tabular}




\section{CONCLUSION}

This study only clustered data rather than classification and after calculating, the FCM method succeeded in conducting data clustering according to the weight of the bipolar wave values, age and heart rate of the patient to determine the 1 st or 2 nd cluster. The test results show that out of 30 patient data tests, as many as 2 patients entered into cluster 1 and as many as 28 patients entered into cluster 2 .

\section{REFERENCE}

[1] D. candra rini Novitasari, Muhammad Fahrur Rozi, and Rafika Veriani, "Klasifikasi Kelainan Pada Jantung Melalui Citra Iris Mata Menggunakan Fuzzy C-Means Sebagai Pengambil Fitur Iris Dan Klasifikasi Menggunakan Support Vector Machine | novitasari | INTEGER: Journal of Information Technology," INTEGER J. Inf. Technol., vol. 4, no. 1, 2019.

[2] Y. J. Indonesia, "No Title.".

[3] J. Nayak, B. Naik, D. P. Kanungo, and H. S. Behera, "A hybrid elicit teaching learning based optimization with fuzzy c-means (ETLBO-FCM) algorithm for data clustering," Ain Shams Eng. J., 2018.

[4] A. K. Muchsin and M. Sudarma, "Penerapan Fuzzy C-Means Untuk Penentuan Besar Uang Kuliah Tunggal Mahasiswa Baru,” Lontar Komput. J. Ilm. Teknol. Inf., 2015.

[5] Muhardi and Nisar, "Penentuan Penerima Beasiswa dengan Algoritma Fuzzy C-Means di Universitas Megow Pak Tulang Bawang,” J. Teknol. Inf. Magister Darmajaya, vol. 01, no. 02, p. 158, 2015.

[6] F. Akbar, "Clustering Data Nilai Siswa SMA Untuk Penentuan Jurusan Menggunakan Algoritma Fuzzy C-Means," vol. 4, no. 1, 2015.

[7] P. Setiaji and W. A. Triyanto, "KLASTERING INDUSTRI DI KABUPATEN KUDUS MENGGUNAKAN METODE FUZZY C-MEANS," Simetris J. Tek. Mesin, Elektro dan Ilmu Komput., 2016.

[8] A. Praja, C. Lubis, and D. E. Herwindiati, "Deteksi Penyakit Diabetes dengan Metode Fuzzy CMeans Clustering dan K-Means Clustering," Comput. J. Comput. Sci. Inf. Syst., 2017.

[9] S. Kusumadewi and Hari Purnomo, Aplikasi Logika Fuzzy untuk pendukung keputusan. Yogyakarta: Graha Ilmu, 2010. 